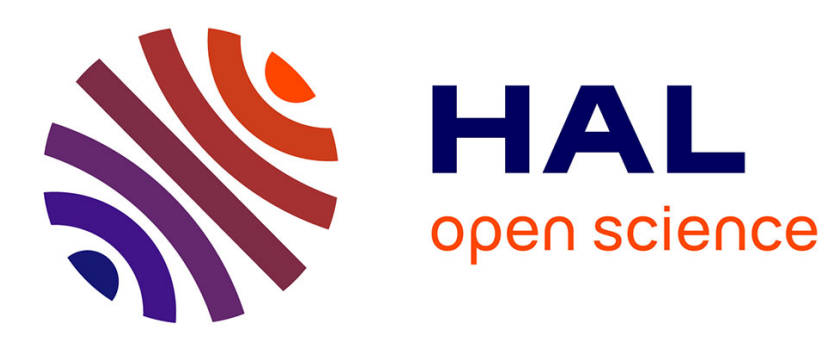

\title{
On the Equilibrium and Welfare Consequences of Getting Ahead of the Smiths
}

Frédéric Gavrel, Thérèse Rebière

\section{To cite this version:}

Frédéric Gavrel, Thérèse Rebière. On the Equilibrium and Welfare Consequences of Getting Ahead of the Smiths. Journal of Public Economic Theory, 2018, 20 (2), pp.257-270. 10.1111/jpet.12261 . halshs-01242504

\section{HAL Id: halshs-01242504 \\ https://shs.hal.science/halshs-01242504}

Submitted on 12 Dec 2015

HAL is a multi-disciplinary open access archive for the deposit and dissemination of scientific research documents, whether they are published or not. The documents may come from teaching and research institutions in France or abroad, or from public or private research centers.
L'archive ouverte pluridisciplinaire HAL, est destinée au dépôt et à la diffusion de documents scientifiques de niveau recherche, publiés ou non, émanant des établissements d'enseignement et de recherche français ou étrangers, des laboratoires publics ou privés. 


\title{
On the Equilibrium and Welfare Consequences of Going Ahead of the Smiths
}

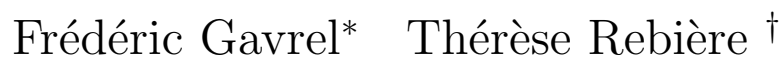

New version, December, 2015

\begin{abstract}
This paper provides an analysis of the social consequences of people seeking to go ahead of the Smiths. All individuals attempt to reach a higher rank than the Smiths, including the Smiths themselves. This attitude gives rise to an equilibrium in which all individuals have equal utilities but unequal (gross) incomes. Due to a rat-race effect, individuals devote too much energy to climbing the social scale. However, laissez-faire equilibrium is an equal-utility constrained social optimum. Conversely, an utilitarian social planner would not choose utility equality. Unexpectedly, this social ambition theory fairly well accounts for empirical intermediate wage inequality.
\end{abstract}

Key words : Going ahead of the Smiths, Social interactions, Well-being, Inequalities, Efficiency.

JEL Classification numbers: D3, D6, D8, I3, Z1.

${ }^{*}$ Corresponding Author, CREM and TEPP, CNRS and Normandy University, Caen, Faculty of Economics and Management, 19 rue Claude Bloch, BP 5186, 14032 Caen Cedex, France; frederic.gavrel@unicaen.fr

${ }^{\dagger}$ LIRSA-CNAM and IZA, Department of Economics, 40 rue des Jeûneurs, Case 1D2P30, 75002 Paris, France; therese.rebiere@cnam.fr 


\section{Introduction}

"So far as concerns the present question, the end sought by accumulation is to rank high in comparison with the rest of the community in point of pecuniary strength." - Veblen (1899)

In this paper, inspired by Veblen (1899), we provide an analysis of the social consequences of people seeking to Go Ahead of the Smiths, GAS henceforth. In accordance with Veblen (1899) ${ }^{1}$, individuals' utilities not only depend on their incomes but also on their social "status". All people attempt to reach a higher social status than the Smiths, including the Smiths themselves. It emerges that the GAS attitude is capable of generating equilibrium income dispersion across ex ante homogenous agents, thus accounting for part of income inequality.

The idea that the individual well-being is to some extent relative to that of others and that we all try to keep up with wealthier than us dates back to Smith (1776) who noticed that happiness is not linked to the stock of acquisitions but to the progressive state of acquiring (see Book I, chapter VIII: of the wages of Labour, paragraph 42). Smith also pointed out that prosperity makes the poorest incapable of being content with the consumption which had formerly satisfied them (see Book I, chapter VIII: of the wages of Labour, paragraph 34). Duesenberry (1949) was the first to empirically observe this relative income phenomenon. The well-know Easterlin paradox (Easterlin 1974) tells us that while the progressive state of income acquisition is correlated with happiness, increased income does not lead itself to increased happiness. As a consequence of this transitory effect of income on life satisfaction, which acts like a focusing illusion (Kahneman et al. 2006), the social concerns behavior leads to a rat-race in which the individual's goal is to place her or himself higher in the income hierarchy.

Our model can be compared with James Mirrlees' path-breaking paper. Contrary to Mirrlees (1971), individuals are identical ex ante. But, since they wish to become VIP's, they turn out to be heterogenous ex post with VIP's consuming a great deal of energy in keeping their envied social position, common people having a low status

\footnotetext{
${ }^{1}$ See Arrow (1975) for a view of Veblen as an economic theorist.
} 
not consuming as much energy in pursuing that end. This gives rise to an equilibrium in which all individuals have equal utilities but unequal (gross) incomes. We derive other interesting insights in this essay. First, we show that there is a single utilitarian social optimum, relative to which all effort is lower than in the case of laissez-faire. This is the consequence of individuals devoting their energy to climbing the social scale. Next, we show that this social optimum generates true inequalities, that is, inequalities in terms of utilities. The reason for this is that a social planner takes advantage of the dispersion of individuals on the ranking scale. However, we find that GAS equilibrium is an egalitarian optimum. It follows that if "you" dislike true inequalities, that is inequalities in terms of utility, then "you" should favor observed (gross) income inequalities, and "you" should firmly reject the idea of any redistributive tax policy. Finally, we show that market equilibrium is inefficient. Pareto inefficiency is the price to pay for true equality.

We also give an assessment of the empirical potential of GAS theory. Unexpectedly, the estimated income distribution rather correctly replicates the observed distribution of wages in France, within an extensive "middle class". Our GAS model can be empirically summarized in two parameters. The first one, referred to as GAS propensity, reflects individuals' desire for advantageous income inequality, while, the other one, referred to as differentiation capability, measures their technological ability to differentiate each other by choosing different effort levels. Using our estimation as a benchmark, numerical simulations describe the effects of GAS propensity and of differentiation capability on income inequality.

The envy/pride hypothesis is indirectly confirmed in the empirical investigations by Easterlin, who finds that "growth does not buy happiness". This unfortunate outcome can be interpreted as a consequence of GAS attitude. If all incomes increase by the same rate, income hierarchy is not affected. Consequently, individuals do not perceive any improvement (in their social status). In that regard, we would like to stress that the analytical tool that we construct in the following assumes rational expectations in the sense that individuals' decisions generate the income distribution on which they base their calculations. Although rational expectations became a nec plus ultra in economic theory about forty years ago, it seems that myopic or adaptative expectations à la Milton Friedman might better account for observed 
facts in the so-called empirical economics of Happiness. Relative concerns have been broadly empirically tested trough well-being sample surveys and experiments since the 90s, see for instance Clark and Oswald (1996), or more recently Card et al. (2012). Indeed, myopic individuals would realize ex post that, despite their efforts, they did not succeed in reaching a higher status since everyone else did too. In other words, while the Smiths were attempting to keep up with the Joneses or go ahead of them, the Joneses themselves moved towards the Harpers who, in turn, moved towards the Rebieres... As a result, the Smiths, Joneses, Harpers and Rebieres, and everybody else report that their happiness is either equal or even falling when asked by Happiness economists.

To position our contribution in the literature $2^{2}$ it is convenient to clearly distinguish between two behavioral issues? ${ }^{3}$. The first issue is: what motivates wealth accumulation? As clearly expressed in the introductory quote, the end sought by accumulation is to rank high in comparison with the rest of the community. This is the social competition argument which we here develop. The second issue is: why do we observe so large expenses in apparently useless items? An answer is that these expenses make wealth visible. We refer to this answer as the signalling argument. Papers like Hopkins and Korienko (2004) or Ireland (2001) retain the "ordinal" version of social concerns. As in the present paper, individuals are affected by their rank in the income hierarchy. In our terminology, they are driven by the GAS attitude. In these papers, contrary to ours, incomes are private information. These papers thus belong to the signalling branch in which agents must be heterogenous. Relative to this strand of literature, our specification of preferences is more restrictive. The reason is that, in an empirical perspective, we have to derive an explicit solution to our GAS model.

Contrary to our contribution, many papers follow the "cardinal" tradition. Similar to Duesenberry, individuals' utilities are affected by the gap between their own "conspicuous" consumption and the average in the population. The cardinal version of social concerns is also referred to as the KUJ (Keeping Up with the Joneses) attitude. To our knowledge, in these papers, information is always perfect. Conse-

\footnotetext{
${ }^{2}$ See Hopkins (2008) for a survey.

${ }^{3}$ We thank Ed Hopkins for very useful comments on the pros and cons of this behavioral literature.
} 
quently, these papers belong to the social competition branch, in which agents can be homogenous. Friedman and Ostrov (2008) develop a very nice study of the dynamics of conspicuous consumption. They also show that social competition may generate ex post heterogeneities among ex ante homogenous agents. When individuals only care about people whose conspicuous consumption is lower - the "pride" hypothesis -, the underlying static model possesses a stable dispersed equilibrium. In general, KUJ applies to the consumption of a positional good but KUJ can also apply to income (models have the same structure) or to real assets as in Cahuc and Postel-Vinay (2005) who develop a behavioral growth model. In a very recent paper where agents are ex ante heterogeneous, Ulph (2014) shows that well-being could decrease with wage rate for those workers who would rather have chosen not to work in the absence of a KUJ behavior.

To summarize, to our best knowledge, earlier papers either develop the social competition argument in the KUJ environment or the signalling argument in the GAS environment. We develop a competition model in the GAS environment. Although both versions are admissible, the GAS assumption sounds more attractive than KUJ whose behavioral foundations are not so clear. In addition, relative to Friedman and Ostrov (2008) in which ex post heterogeneities result from the "pride" version of KUJ hypothesis, we find that the GAS attitude necessarily gives rise to income inequality, even though agents are ex ante homogenous.

This insight is close in spirit to Burdett and Judd (1983) for example where equilibrium price dispersion does not result from some ex ante heterogeneity of agents but only from non-sequential search. Another example is Burdett and Mortensen (1998) where the same holds for the equilibrium dispersion of wages which entirely stems from on-the-job search. In other words, we exhibit an income dispersion (i.e inequality) equilibrium which entirely results from the GAS behavior.

Section 2 builds our modeling of GAS equilibrium. The welfare implications of social ambition are studied in section 3 while section 4 examines the empirical potential of GAS model. The conclusion summarizes our findings and sets a non-exhaustive agenda for further investigations. 


\section{Environment and GAS equilibrium}

Let us consider an environment à la James Mirrlees. Following this author, individuals' income, $y$, is an increasing function of their effort, $k$. But, contrary to Mirrlees (1971), all individuals have the same efficiency at work, implying that they are perfectly identical ex ante. In other words, there is a single technology $y=H(k)$, with $H(k)$ being an increasing (strictly) concave function which satisfies $H(0)>0.4$ The set of individuals is a continuum whose measure is normalized to 1 .

Individuals' investments generate an endogenous c.d.f., $\Pi(k)$, which represents the proportion of individuals whose investments are strictly lower than $k$, i.e. $\Pi(k)$ $=$ Prob $[$ Effort $<k]$. This definition of $\Pi($.$) makes the exposition easier. A priori$ this function is assumed to be piecewise continuous. Its mass points are denoted by $\left(K_{1}<\ldots<K_{i}<\ldots<K_{n}\right)$ where the positive integer $n$ can be unbounded. The corresponding frequencies are $\left(\phi\left(K_{1}\right), \ldots, \phi\left(K_{n}\right)\right)$. If $n$ is zero, $\Pi($.$) is continuous on$ $[0, \infty[$.

These people are envious. They are all willing to go ahead of the Smiths, implying that they are affected by their location in the income scale. In this context, the utility of an individual, $U(k,$.$) , is decreasing in her effort, while increasing in her rank in$ the income hierarchy as well as in her output. In an empirical perspective, we need to derive an explicit solution. In this spirit, we retain the following specification

$$
U(k, .)=-k+J(\Pi(k)) H(k)
$$

In the previous expression, the term $J($.$) , referred to as the "GAS multiplier", is$ a strictly increasing (continuous) function of the share, $\Pi(k)$, of strictly lower ranked participants:5 It is worth noting that, according to our definition, $\Pi(k)=\Pi\left(k^{-}\right)$. Thus, if $K_{i}$ is a mass point, $\Pi\left(K_{i}^{+}\right)=\Pi\left(K_{i}\right)+\Phi\left(K_{i}\right)$. With no loss of generality, the top, $J(1)$, can be set to 1 while the bottom, $J(0)$, assumed to be strictly positive, is denoted by $\mu(0<\mu<1)$.

This specification of utility $U($.$) nicely captures the ambition of Going Ahead of$

\footnotetext{
${ }^{4}$ This (reasonable) assumption makes the analysis simpler.

${ }^{5}$ Results extend to the case in which the multiplier associated with a mass point $K_{i}$ is higher than $J\left(\Pi\left(K_{i}\right)\right)$ but lower than $J\left(\Pi\left(K_{i}\right)+\Phi\left(K_{i}\right)\right)$.
} 
the Smiths. It is not additiv ${ }^{6}$, implying that the downgrading (or upgrading) effect is all the higher as individuals are rich. This is in conformity with Thorstein Veblen's message (1899). It is worth noting that individuals only care about lower ranked people. The reason is that, contrary to the cardinal version of social concerns, the ordinal version does not allow for distinguishing between higher and lower ranked players. More rigorously, this distinction can be made but has not any formal incidence. Indeed, suppose that instead of $\Pi(k)$, individuals care about the weighted average $\alpha \Pi(k)-(1-\alpha)(1-\Pi(k))$ with $(0 \leq \alpha \leq 1)$. In the terminology of Friedman and Ostrov (2008), the term $\alpha \Pi(k)$ translates a "pride" feeling while a "frustration" feeling is expressed through the term $-(1-\alpha)(1-\Pi(k)$. As $\alpha \Pi(k)-(1-\alpha)(1-\Pi(k))$ $=\Pi(k)-(1-\alpha)$, this distinction, which clearly has a behavioral content, has not any analytical content.

Each individual maximizes $U(k)$ with respect to her effort $k$ for a given c.d.f. $\Pi(k)$. It is also assumed that function $V(k)=-k+H(k)$ goes to $-\infty$ as $k$ goes to $+\infty$ and that the derivative of $V(k)$ is strictly positive for $k=0$. This implies that $V($.$) has a single maximum \tilde{k}>0$ with $V(\tilde{k})=\tilde{V}$. On the other hand, function $W(k)$ denotes $-k+\mu H(k)$.

In this environment an equilibrium can be defined as below.

Definition 1 A GAS equilibrium is a distribution function $\Pi^{*}($.$) such that, for all k$ in its support, $U(k)$ is maximized with respect to $k \geq 0$.

It can be noted that this definition implies that $U(k)$ is a constant $U^{*} \geq U(0)>0$, for all $k$ in the support of an equilibrium distribution. Let us study this equilibrium.

In Appendix A, it is proved that the equilibrium distribution is continuous and that its support, denoted by $S^{*}$, is a bounded interval $[A, B]$ with $0 \leq A<B<\infty$. For simplicity, the analysis focus on the case in which the lower bound, $A$, is strictly positive. In $S^{*}, U(k)$ is a constant $U^{*}>0$. Consider the behavior of the GAS multiplier $J(k)=J(\Pi(k))$ in the interval $[A, B]$. We have $J(A)=\mu$. In the interval

\footnotetext{
${ }^{6}$ Additive relative concerns would make technological progress incompatible with steady income inequality.
} 
$[A, B], J(k)$ is strictly increasing and equal to one for $k=B$. In this interval, $J(k)$ satisfies (see equation (11)

$$
J(k)=\frac{k+U^{*}}{H(k)}
$$

So $J(k)$ has a derivative $J^{\prime}(k)$ such that

$$
J^{\prime}(k)=\frac{H(k)-\left(k+U^{*}\right) H^{\prime}(k)}{H(k)^{2}}
$$

One can check that if $J^{\prime}(A) \geq 0$, then $J^{\prime}(k)>0$ in $\left.] A, B\right]$. As $U(A)$ should be equal to $U^{*}$, it follows that $J^{\prime}(A) H(A)=1-\mu H^{\prime}(A) \geq 0$. Consider the behavior of $U(k)$ in $[0, A[$. In this interval, $U(k)=W(k)$ should be lower than $U(A)$. The concavity of $H($.$) then imposes that -1+\mu H^{\prime}(A) \geq 0$. See Figure 1. It results that $-1+\mu H^{\prime}(A)=0$. Consequently, $S^{*}$ is an interval $[A, B]$ whose lower bound is determined by $-1+\mu H^{\prime}(A)=0$. The latter equation has a solution $A^{*}(>0)$ if and only if $-1+\mu H^{\prime}(0)>0$. From $A^{*}$, we deduce $U^{*}=U\left(A^{*}\right)$.

Let us now turn to the upper bound. Since $\Pi^{*}(B)=1, B^{*}$ satisfies $-B+H(B)=$ $U\left(A^{*}\right)$. For $k>B^{*}, U(k)=V(k)=-k+H(k)$. This implies that $B^{*} \geq \tilde{k}$. If not, $U(k)=V(k)$ would be strictly increasing for $B^{*}<k<\tilde{k}$. Consequently, $B^{*}$ is the highest solution to $-B+H(B)=U\left(A^{*}\right)$. See Figure 1 .

Results are summarized in the proposition below.

Proposition 1 If and only if $-1+\mu H^{\prime}(0)>0$, the support of $\Pi^{*}($.$) is an interval$ $\left[A^{*}, B^{*}\right]$ with $0<A^{*}<B^{*}$. $A^{*}$ is the solution to $-1+\mu H^{\prime}(A)=0$ while $B^{*}$ is the highest solution to $-B+H(B)=U^{*}=U\left(A^{*}\right)$. In this interval, GAS equilibrium satisfies $\Pi^{*}(k)=J^{-1}\left(\left(k+U^{*}\right) / H(k)\right)$ ]

In fact, the derivation of a GAS equilibrium is simple. The investment of individuals at the bottom of hierarchy does not depend on the investment distribution. And the same holds for individuals at the top. Between the two extremes, $\Pi^{*}(k)$ is set so

\footnotetext{
${ }^{7}$ One can see that if $-1+\mu H^{\prime}(0) \leq 0$, then $S^{*}$ is an interval $\left[0, B^{*}\right]$, with $B^{*}$ being the highest solution to $-B+H(B)=\mu H(0)$.
} 
as to ensure that utilities are constant and equal to $U\left(A^{*}\right)$. From $\Pi^{*}(k)=1$, hence, $J(k)=1$, one deduces the upper bound of the support (which should be greater than the lower bound).

Figure 1 illustrates the determination of an equilibrium. As $W(k)$ is maximized in $A^{*}, U(k)$ coincides with $W(k)$ for $0 \leq k \leq A^{*}$, is equal to $U^{*}\left(=U\left(A^{*}\right)\right)$, for $A^{*} \leq k \leq B^{*}$, and coincides with $V(k)$, beyond $B^{*}$.

Figure 1: GAS Equilibrium, $W^{\prime}(0)>0$.

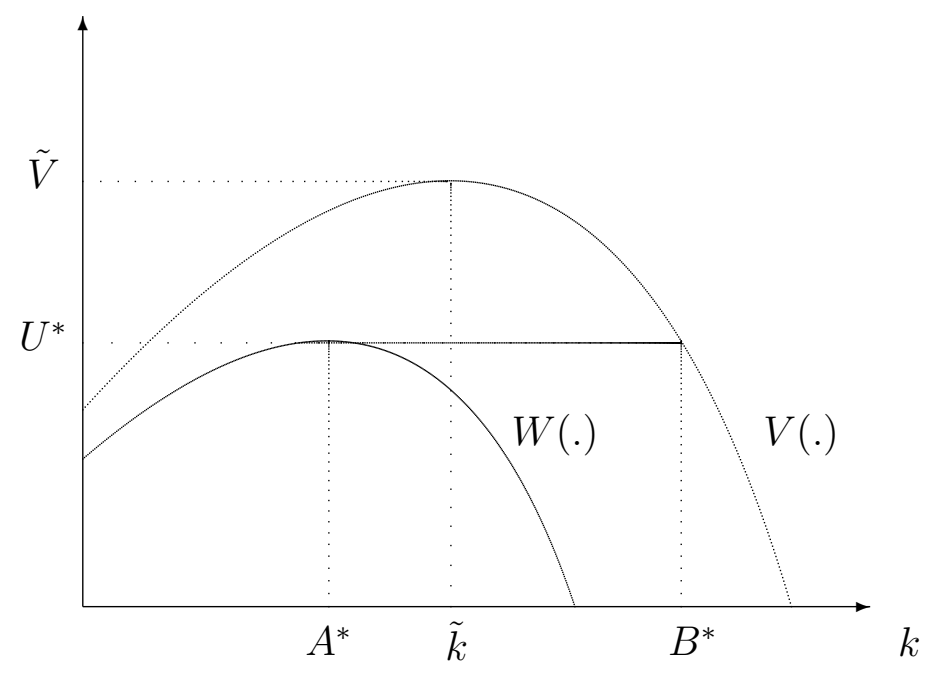

Individuals are identical ex ante. But, since they wish to become VIP's, they turn out to be heterogenous ex post, with VIP's consuming a great deal of energy in keeping their envied social position, common people having a low status not consuming as much energy to pursuing that end. This gives rise to an equilibrium in which all individuals have equal utilities, but unequal (gross) incomes. Income inequality is not very surprising. Indeed, starting from a situation of income equality, a (very) small increase in effort is sufficient to reach the top of the social hierarchy, implying that such a symmetric outcome cannot be an equilibrium. On the other hand, one could predict that all individuals will be prompted to "do the maximum". This is what can occur when beyond some ceiling, the marginal return to effort is reduced to 
zero. It can be noted that equilibrium existence is ensured for all strictly increasing GAS multiplier, $J(\Pi(k))$. Numerical simulations given in section 4 throw some light on the comparative statics.

\section{$3 \quad$ Welfare analysis}

In this section, we study the welfare properties of laissez-faire equilibrium in relation to different criteria. We find that a decentralized equilibrium is not an utilitarian optimum. The reason is that a social planner would take advantage of the income hierarchy. Interestingly, laissez-faire generates an egalitarian optimum. Which means that market equilibrium maximizes the utilitarian criterion subject to the constraint of utility equality. To conclude, it is proved that decentralized equilibrium is inefficient in the sense of Pareto. This stresses the price to pay for true equality (equality in terms of utilities).

\section{$3.1 \quad$ Utilitarian criterion}

Following the utilitarian tradition, the welfare criterion is the weighted average of utilities. Let $S(\Pi()$.$) denote the support of \Pi($.$) . Social welfare is$

$$
\Sigma(\Pi(.))=-\int_{S(\Pi(.))} k d \Pi(k)+\int_{S(\Pi(.))} J(\Pi(k)) H(k) d \Pi(k)
$$

To characterize the social optimum, we rank the individuals uniformly (and continuously) on the segment $[0,1]$. As the ranking is uniform, there is a share $r$ of individuals whose ranks are lower than $r$ (i.e. whose ranks lie in the interval $[0, r]$ ). Making use of this ranking model of effort dispersion, $[k(r)]_{0}^{1}$, denotes the effort of an individual whose rank in the income scale is $r(0 \leq r \leq 1)$. The effort function, $k($.$) ,$ is the reciprocal of the distribution function $\Pi($.$) (when it exists). Accordingly, the$ welfare criterion can be rewritten as

$$
\Sigma\left([k(r)]_{0}^{1}\right)=-\int_{0}^{1} k(r) d r+\int_{0}^{1} J(r) H(k(r) d r
$$

An utilitarian social optimum, denoted by $\left[k^{S}(r)\right]_{0}^{1}$, maximizes the welfare criterion 
with respect to $[k(r)]_{0}^{1}$. Let $k^{*}(r)$ denote the reciprocal of laissez-faire equilibrium, $\Pi^{*}(k)$. We can prove the following

Proposition 2 (i) According to an utilitarian planner, GAS model has a single social optimum, $\left[k^{S}(r)\right]_{0}^{1}$, such that $-1+J(r)(d H(k(r)) / d k(r))=0$ for all $r$ in $[0,1]$. $k^{*}(r)>k^{S}(r)$ for all $r$ in $[0,1]$.

From the ranking optimum $k^{S}(r)$, we deduce the optimum distribution $\Pi^{S}(k)$ which is the reciprocal of $k^{S}(r)$.

Statement (i) results from the fact that the two models of effort dispersion, either the distribution $\Pi(k)$, or the ranking function $k(r)$, are isomorphic. See Appendix B. In words, deciding on a non-decreasing function $k(r)$ is not different from deciding on a distribution function $\Pi(k)$. 8 So the proof of statement (i) amounts to observing that, due to the concavity of $H(),. k^{S}(r)$ is strictly increasing in $r$. At first glance this result might look counter-intuitive, however. Indeed, one could object that, if defined with a c.d.f. $\Pi(k)$, a social optimum might have mass points. This can be excluded. The reason is that any mass point $K_{i}$ of $\Pi(k)$ corresponds to a subinterval of $[0,1]$ in which $k(r)$ is constant and equal to $K_{i}$. Consequently, any mass point generates a constraint on $[k(r)]_{0}^{1}$. Since $J(r)$ is strictly increasing, such constraints would be binding for the social problem. Symmetrically, one can easily exclude the case in which the social optimum defined as a distribution $\Pi^{S}(k)$ would be constant in a subinterval of $\left[A^{S}=k^{S}(0), B^{S}=k^{S}(1)\right]$. This is because the ranking of individuals on $[0,1]$ would create a discontinuity of $k^{S}(r)$ in such a case. Hence, the social optimum, $\Pi^{S}(k)$, which is unique, is also continuous and strictly increasing in the interval $\left[A^{S}, B^{S}\right]$. See Appendix B and C.

Statement (ii) results from the strict concavity of $-k(r)+J(r) H(k(r))($ in $k(r))$. In words, this result means that, relative to the social optimum, laissez-faire generates too much investment. In a precise sense, that is, for all $r$ in $[0,1]$. As state-

\footnotetext{
${ }^{8}$ If $\mathrm{X}$ is a random variable whose c.d.f. $\mathrm{F}($.$) is continuous and strictly increasing, then the random$ variable $\mathrm{Y}=\mathrm{F}(\mathrm{X})$ is uniformly distributed on $(0,1)$. The proof of Proposition 3 just generalizes this property to a piecewise continuous non-decreasing function $\mathrm{F}($.$) , then allowing for mass points.$
} 
ment (i) makes clear, this comes from the ranking effect, measured by the term $d J(\Pi(k) / d k) H(k)$. In GAS language, this result is the consequence of individuals devoting their energy $k$ to climb up the social scale, aiming at having more people below them, or, equivalently, fewer people above them. This generates a rat-race effect which makes individuals' efforts excessive. On the contrary, a social planner would decide on investments $[k(r)]_{0}^{1}$ for a given ranking of individuals.

One implication of statement (i) is interesting per se. It turns out that, in the social optimum, the utility $U(r)=U\left(k^{S}(r)\right.$ ) (whose derivative reduces to $U^{\prime}(r)=$ $\left.J^{\prime}(r) H\left(k^{S}(r)\right)>0\right)$ is strictly increasing. By this path, we have thus reached the result that, in this context, although individuals are homogenous ex ante, utility inequality is better for welfare. This is an unexpected outcome, since a priori, one could surmise that, due to the concavity of $H($.$) , the social optimum should be$ symmetric. As the ranking model makes clear, a social planner takes advantage of the dispersion of individuals across the ranking scale. The reason is that the marginal product of effort grows with the individuals' position in the hierarchy. In other words, rich people profit more from an increase in their (gross of effort) revenue. Or, equivalently, people are increasingly sensitive to social degrees when their income is higher. Consequently, utilitarian welfare is higher with a hierarchy of individuals.

It is worth noticing that Proposition 2 dramatically depends on the definition of aggregate welfare. A (virtual) situation in which true (i.e. utility) inequality would be favored because "rich people should be very rich" is a bit shocking. Extending the analysis to other welfare criteria is an interesting line for further investigations. At this stage, we can ask the following question. Which income distribution would an egalitarian planner select? We can prove that GAS equilibrium maximizes the utilitarian criterion subject to the constraint of utility equality. In other words

Proposition 3. According to an egalitarian planner, GAS equilibrium is a social optimum.

The analysis can be restricted to a continuous distribution whose support is connected $9^{9}$ In this case, the proof of Proposition 4 consists in observing that the equal-

\footnotetext{
${ }^{9}$ Mass points of probability (strictly) lower than one are clearly incompatible with the equal-
} 
utility constraint $U^{\prime}(r)=0$ implies that, for all $r$ in $[0,1]$,

$$
-k^{\prime}(r)+J(r) H^{\prime}(k(r)) k^{\prime}(r)+J^{\prime}(r) H(k(r))=0
$$

or,

$$
-1+J(k(r)) H^{\prime}(k(r))+\frac{d J(\Pi(k(r)))}{d k(r)} H(k(r))=0
$$

The latter condition is satisfied by GAS equilibrium $\Pi^{*}(k)$. It follows that this (strictly) egalitarian social planner only has a single degree of freedom which is used to make $U(0)=-k(0)+\mu H(k(0))$ as high as possible. This maximum is reached for $k(0)=A^{*}$. Since $k(r)$ cannot be decreasing, it results that $k(1)=B^{*} 10$ This shows that GAS equilibrium is an equal-utility constrained social optimum.

The implications of Proposition 3 can be expressed in the following maxima: Whoever dislikes true inequalities, that is inequalities in terms of utility, should like observed (gross) income inequalities, and should then fight against any redistributive tax policy. It is worth noting that "true" equality (in terms of utilities) is particularly relevant in an economy where individuals are identical.

\subsection{Pareto criterion}

Let us finally prove that an equilibrium is Pareto inefficient: all participants invest too much in the neighborhood of GAS equilibrium.

In market equilibrium $\Pi^{*}($.$) , the effort of r$-workers satisfies $r=\Pi^{*}(k)$ or $k=$ $k^{*}(r)$, with $k^{*}($.$) being the reciprocal of \Pi^{*}($.$) . The equilibrium utility of an individual$ with rank $r$ is

$$
-k^{*}(r)+J(r) H\left(k^{*}(r)=-k+J\left(\Pi^{*}(k)\right) H(k)\right.
$$

Making use of this ranking of individuals, let us study the effect of a small change $d K$ in all efforts in the neighborhood of equilibrium. Since all investments vary

utility constraint. The same holds for holes in the support which are mapped into a discontinuity of $k(r)$ (see Appendix B). Conversely, one can see that the equal-income social optimum is another equal-utility constrained social optimum.

${ }^{10}$ Notice that the derivative $d J(\Pi(k(r))) / d k(r)$ is equal to zero for $r=0$ but $J^{\prime}(r)>0$. This is because, for $r=0$, $d J(\Pi(k(r))) / d k(r)$ is zero but, since $r=\Pi(k(r)),(d \Pi(k(r)) / d k(r)) k^{\prime}(r)=1$ for all $r$ in $[0,1]$. 
by the same amount, the rank $(r)$ associated with $\left(k^{*}(r)+d K\right)$ is left unchanged. Consequently, the change in the utility of an agent of rank $r$ is

$$
d U(r)=\left[-1+J(r) \frac{d H\left(k^{*}(r)\right)}{d k(r)}\right] d K
$$

On the other hand, in GAS equilibrium, all individuals have the same utility $U^{*}$, implying that

$$
-1+J(r) \frac{d H\left(k^{*}(r)\right)}{d k(r)}=-\frac{d J\left(\Pi^{*}\left(k^{*}(r)\right)\right)}{d k(r)} H\left(k^{*}(r)\right)
$$

We then obtain

$$
\frac{d U(r)}{d K}=-\frac{d J\left(\Pi^{*}\left(k^{*}(r)\right)\right)}{d k(r)} H\left(k^{*}(r)\right)<0
$$

This proves that 11

Proposition 4 According to a Paretian planner, in the neighborhood of GAS equilibrium, lowering individuals' efforts improves welfare.

This result clearly comes from the GAS hypothesis. A decrease in all investments (i.e. efforts), which does not affect the position of agents, increase all utilities. The same ranks are reached at a lower "price". Cooperation could enhance welfare in the sense of Pareto 12

This insight stresses the cost to pay for egalitarian efficiency. Lowering all efforts is Pareto-improving but individuals enjoy different utility gains. Assuming that GAS multiplier $J(r)$ is convex, one can see that the higher the rank, the higher the marginal utility gain $\left(J^{\prime}(r) H(k(r))\right.$. These disparities are not compatible with egalitarian efficiency.

\footnotetext{
${ }^{11}$ Provided that they keep in the same position, all individuals enjoy a utility gain.

${ }^{12}$ Notice that Proposition 4 obviously implies that market equilibrium is not an utilitarian optimum. On the contrary, Pareto efficiency does not imply utilitarian efficiency.
} 


\section{Empirical potential and predictions of GAS the- ory}

Can GAS equilibrium replicate observed wage dispersion? This section shows that GAS equilibrium fairly well accounts for the empirical wage distribution in France. How does a change in GAS attitude affect income inequality? We also carry out numerical simulations aimed at throwing some light on different issues.

The specification for the GAS multiplier is borrowed to urn-ball matching with recruitment selection (see Gavrel 2012, 2015). That is

$$
J(r)=\exp (-\lambda(1-r))
$$

In the previous expression for the GAS multiplier, the positive parameter $\lambda$ can be regarded as a measure of individuals' GAS propensity, also referred to as their "social ambition". The higher this parameter, the higher the effect of social rank on individuals' utilities. From section 2 (Proposition 1), we know that the bottom and the top of (gross) income distribution are respectively determined by $H\left(A^{*}\right)$ with $-1+J(0) H^{\prime}\left(A^{*}\right)=0$, and by $H\left(B^{*}\right)$ with $B^{*}$ being the highest solution to $U(B)=U^{*}=U(A)$, or $-B+H(B)=-A^{*}+J(0) H\left(A^{*}\right)$.

We also know that the reciprocal of the equilibrium distribution of investments, $k^{*}(r)(0 \leq r \leq 1)$ can be deduced from

$$
J(r)=\frac{k-U^{*}}{H(k)}
$$

or, in terms of income $y$,

$$
J(r)=\frac{H^{-1}(y)+U^{*}}{y}
$$

In the following calculations, the production function, $H($.$) is specified as H(k)=$ $a+b k^{1 / 2}$ with $a$ and $b$ being strictly positive. Notice that $H^{\prime}(0)=\infty$, implying that $A^{*}>0$, as in GAS equilibrium $\left(W^{\prime}(0)>0\right)$. GAS propensity, $\lambda$, is strictly positive. The equilibrium value of utility, $U^{*}=U\left(A^{*}\right)$ is deduced from 


$$
A^{*}=\frac{(b J(0))^{2}}{4}
$$

It results that

$$
U^{*}=\frac{(b J(0))^{2}}{4}+a J(0)
$$

Let $q$ denote $q=b^{2} / a$. The reciprocal of the cumulative distribution function the income function $y^{*}(r)$ - should satisfy the following second degree equation:

$$
(y / a)^{2}-[2+q J(r)](y / a)+\left[1+q J(0)+\frac{(q J(0))^{2}}{4}\right]=0
$$

It is easy to prove that $y^{*}(r)$ is the highest solution to the former equation. This is because $y(r)$ should be increasing. We obtain the following econometric model:

$$
y(r)=a \frac{2+q J(r)+[q J(r)(4+q J(r))-q J(0)(4+q J(0))]^{1 / 2}}{2}+\epsilon
$$

where $J(r)$ is set by equation (5).

It should be noted that results depend on the units in which wages are measured. In other words, $a$ is a scale parameter. Only coefficient $q$ has an economic meaning. This coefficient measures the extent to which individuals can differentiate each other by deciding on different effort levels. The random variable $\epsilon$ is the error term. This GAS model of income dispersion is estimated by applying non linear least squares. Notice that estimating the reciprocal of the cumulative distribution function allows us to treat the income $y(r)$ associated with rank $r$ as a random variable. In addition, the bounds $z=H\left(A^{*}\right)$ and $Z=H\left(B^{*}\right)$ do not necessarily coincide with the bounds of the observed (truncated) distribution.

\subsection{Empirical potential}

Figure 2 reports the actual distribution of wages in France in 2010. It can be noted that the lower tail of the actual distribution is quite flat. In addition, individuals showing similar behavior should belong to the same "community". For this reason, 
we limit our study on the one hand to the poverty threshold, that is to say to $60 \%$ of the median income ${ }^{13}$, and on the other hand to the 95 th percentile so that monthly gross incomes vary between 1317 and 5586 euros.

Figure 2: Income distribution for France, full distribution

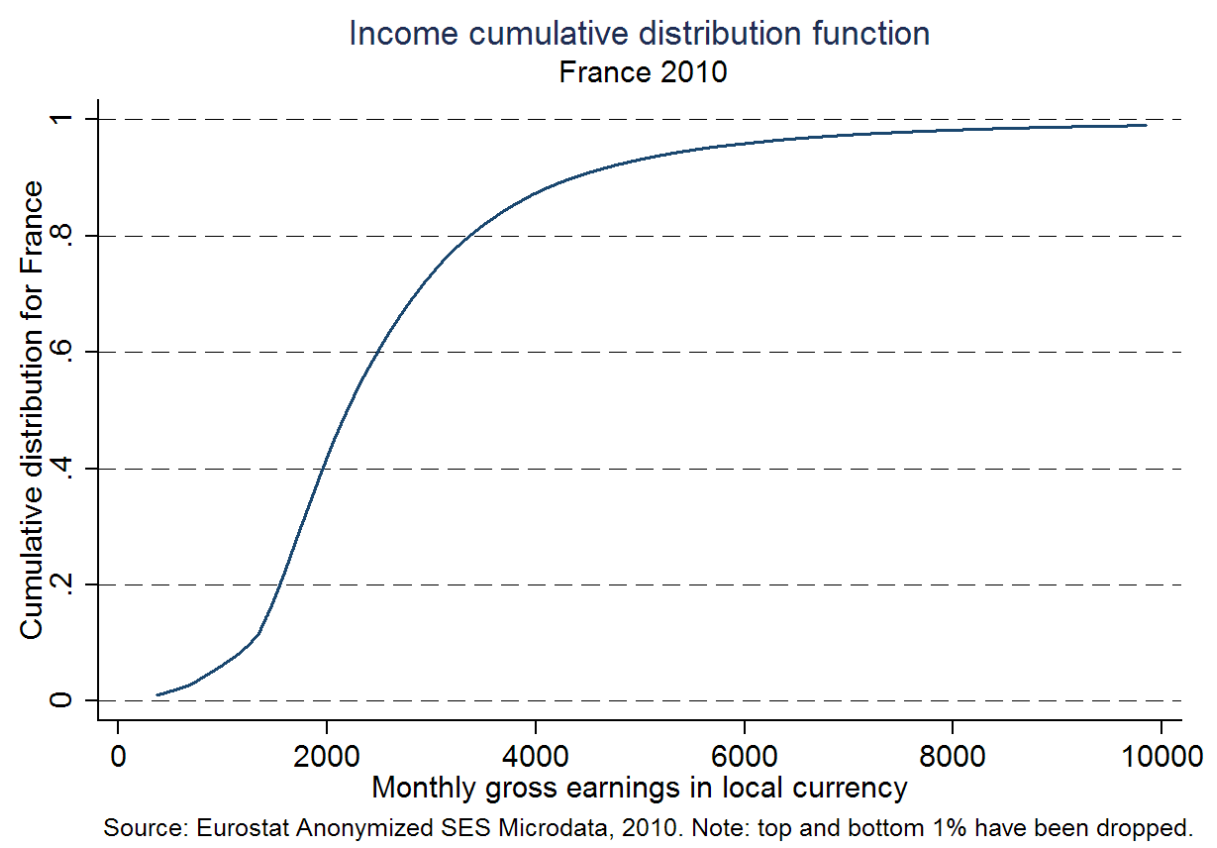

As mentioned above, the estimation of GAS model applies non-linear least squares to the reciprocal $y^{*}(r)$ of the income cumulative distribution. In accordance with The Theory of Leisure Class, which here extends to a large "middle class", the actual truncated wage distribution is rather better replicated by the estimated distribution (see the observed and estimated reciprocal of the income cumulative distribution presented on Figure 3). Table 1 reports the econometric results.

\footnotetext{
${ }^{13}$ This amounts to dropping about $10 \%$ of the bottom of the distribution.
} 
Figure 3: Actual and estimated reciprocal of the income cumulative distribution for France

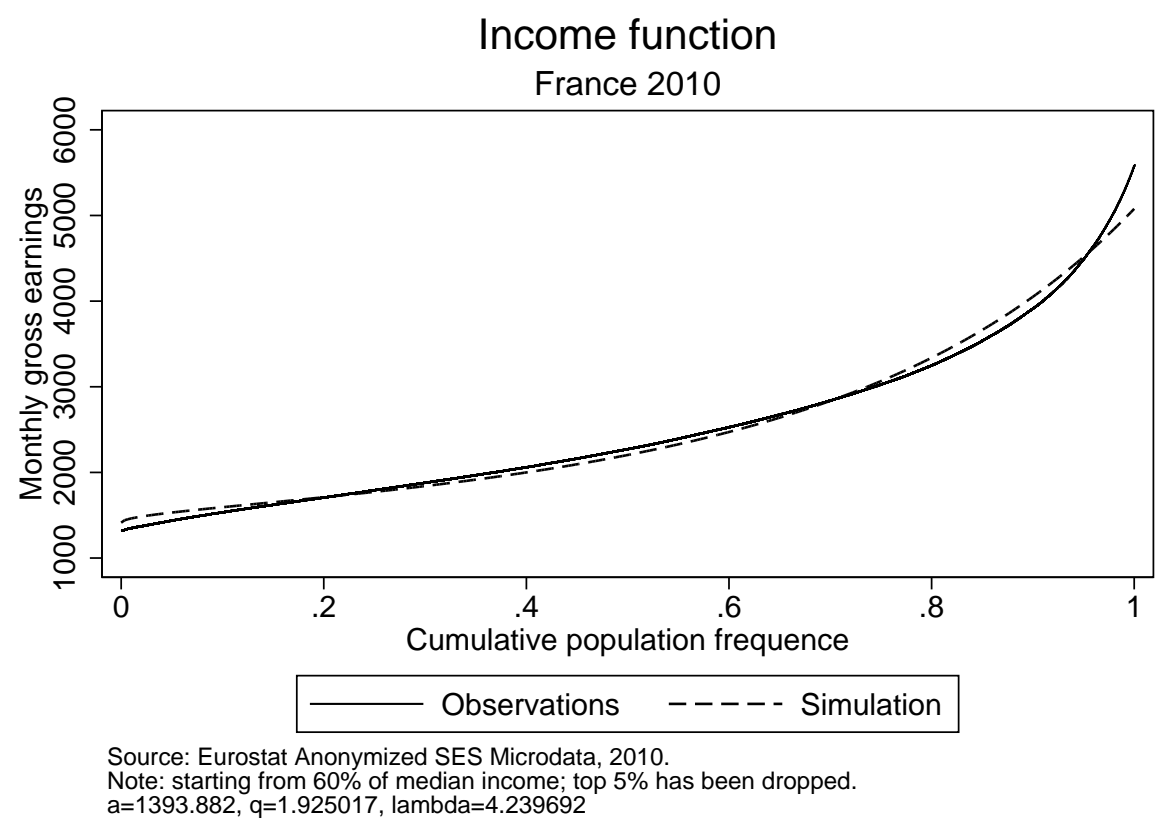

Table 1: OLS estimation of income function

Coef. Std. Err. Number of obs $=184236$

Differentiation capability

$q \quad 1.925017^{* * *} \quad 0.001256$

R-squared $=0.999$

GAS propensity

$\lambda \quad 4.239692^{* * *}$

0.0029779

Scale parameter

a $1393.882^{* * *} \quad 0.5622717$

Figure 4 depicts the empirical Lorenz curve together with the estimated Lorenz curve (deduced from the estimated cumulative distribution). One can notice that the two curves are very close to each other. GAS theory fairly well accounts for intermediate wage inequality. 
Figure 4: Empirical and simulated Lorenz curves

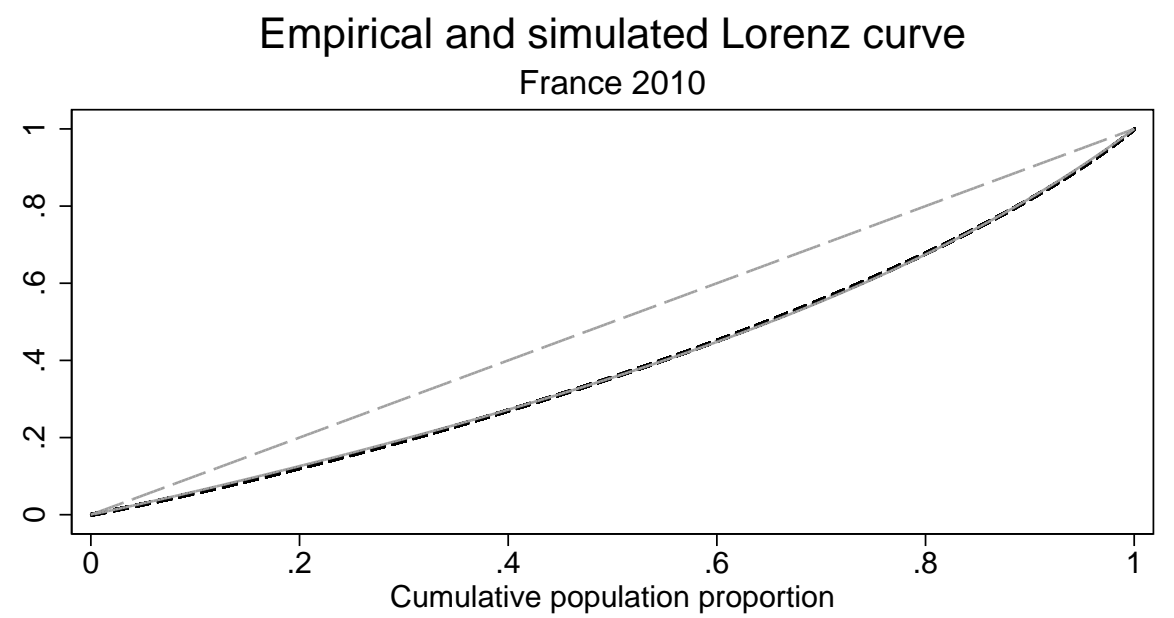

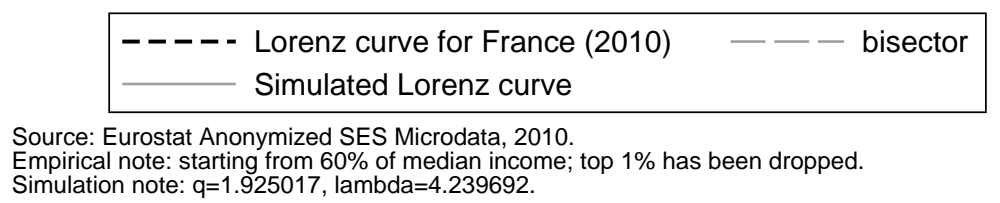

\subsection{Predictions}

Let us first study the impact of GAS propensity on income inequality and on poverty.

Figures 5 shows the simulated Lorenz curves for different values of GAS propensity, $\lambda$, using the estimated value of the differentiation parameter, that is $q=1.925017$. 
Figure 5: Lorenz curve sensitivity to GAS propensity

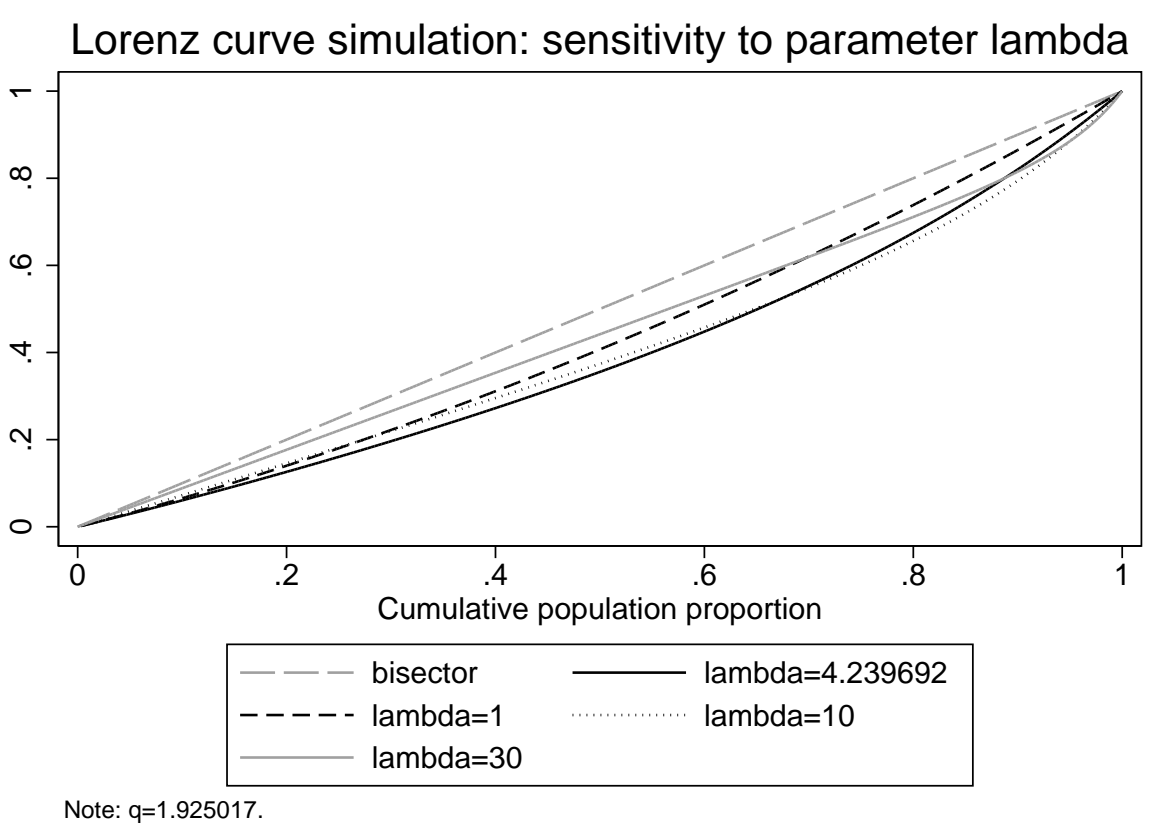

GAS attitude gives rise to inequalities. The intuition behind this is simple. Individuals are homogenous, implying that, in the absence of "social ambition" $(\lambda=0)$, they all earn the same income. In the presence of social ambition, equality becomes impossible, as explained in the comment on Proposition 1. Notice that beyond some threshold, curves intersect: an increase in GAS parameter would tend to lower income inequalities. This is because all efforts go to zero as parameter $\lambda$ goes to infinity. This point can be made clearer by making use of Gini coefficient for inequality. The Gini coefficient of the empirical truncated income distribution is 0.2023696 while the simulation leads to pretty close coefficient of 0.2011106. Figure 6 depicts the Gini coefficient as a function of GAS propensity. Above about $\lambda=6$, an increase in GAS propensity reduces income inequalities. 
Figure 6: Gini sensitivity to GAS propensity $\lambda$

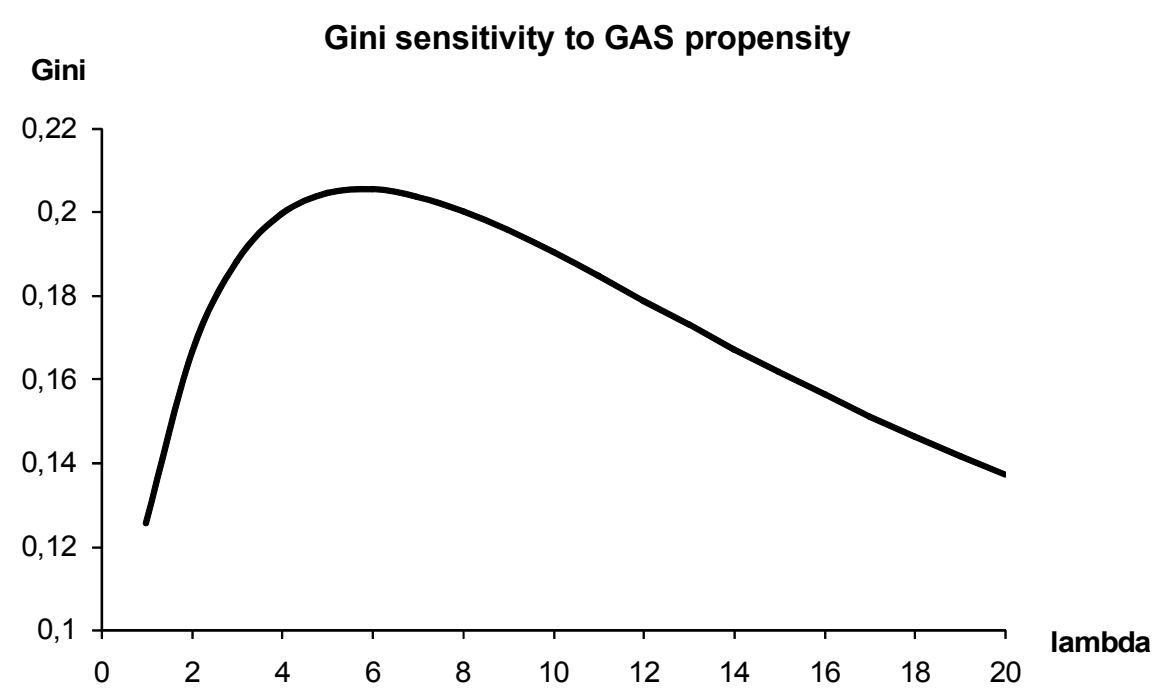

Note: $q=1.925017$.

Besides, one can show that an increase in GAS propensity lowers individuals' utilities ${ }^{14}$. But for any given rank in the income scale, an increase in GAS propensity does not invariably raise (or lower) the corresponding (gross) income (see Figure 7). In other words, the effect on "perceived" wealth (or poverty) is ambiguous.

\footnotetext{
${ }^{14}$ Knowing that all individuals have the same utility in GAS equilibrium, the proof amounts to see that an increase in social ambition (parameter $\lambda$ ) reduces the utility $U^{*}=U\left(A^{*}\right)=-A^{*}+J(0) H\left(A^{*}\right.$ of individuals at the lower end of the social scale. GAS multiplier $J(0)=\exp (-\lambda)$ falls.
} 
Figure 7: Simulated income distribution sensitivity to GAS propensity

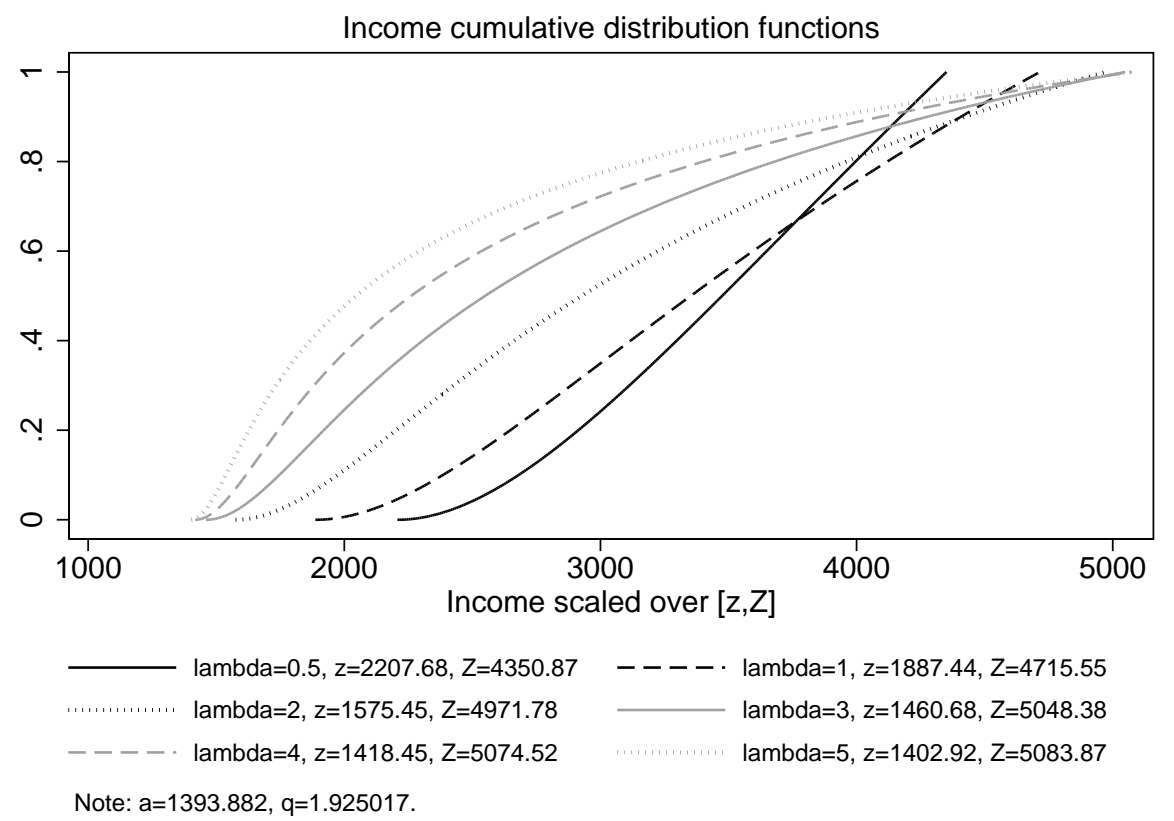

Note: $\mathrm{z}$ and $\mathrm{Z}$ are the inferior bound $\left(H\left(A^{*}\right)\right)$ and superior bound $\left(H\left(B^{*}\right)\right)$.

Another interesting issue is the way in which individuals' ability to differentiate each other - their differentiation capability - affects income inequalities. Holding parameter $a$ as a constant, an increase of parameter $q$ can be seen as a consequence of economic development. 15 Figure 8 describes the effect of such improvement of labor efficiency on "perceived" inequality. In conformity with Veblen's intuition, an increase in labor efficiency would tend to expand income inequality. Indeed, individuals cannot differentiate when labor efficiency is very low.

It can be noted that these comparative statics exercises have very different meanings. In the second case, wealth is affected by a "material" phenomenon whereas, in the first case, the change in utilities and incomes results from a social phenomenon. This highlights the role of social values (like social ambition) in the economic sphere as well as the practical importance that value-oriented policies may have in the real

\footnotetext{
${ }^{15}$ Holding parameter $q$ as a constant, an increase in parameter $a$ leaves income inequality unchanged. In this sense, our specification of preferences makes that technological progress is compatible with steady income inequality.
} 
world. Contrary to K. Marx but in accordance with J.M. Keynes, "ideas" may have economic consequences. They may exert an influence on the so-called economic "infra-structures". This is a domain where "free lunches" could be found, although a marxian economist would probably object that GAS behavior, like individualism, is determined by the economic environment.

Figure 8: Simulated Lorenz curve sensitivity to differentiation capability, $q$

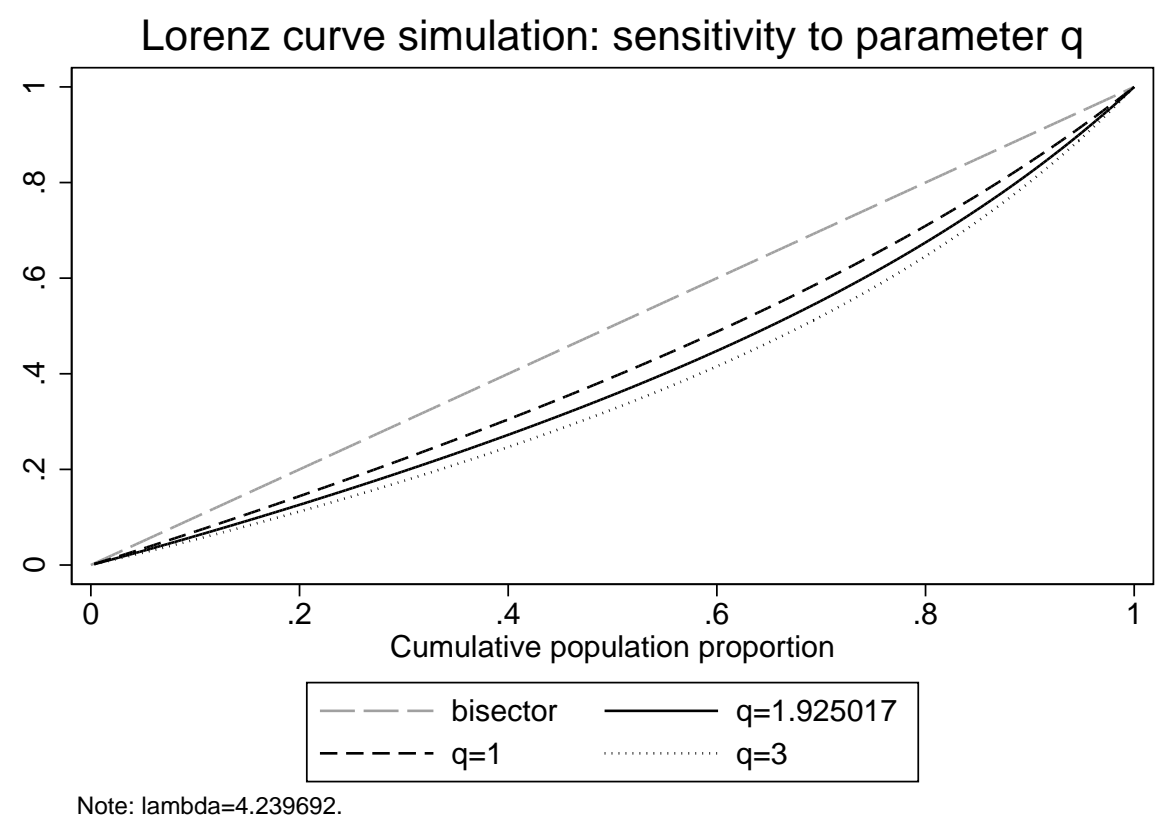

To sum up, these empirical exercises permit us predict that the GAS model does have empirical potential. We acknowledge that, to some extent, GAS theory is difficult to refute, since the plausibility of parameters values cannot be easily assessed. A natural way of circumventing this problem would be to assume that, excepting GAS intensity $\lambda$, other parameters are common to different countries. In that regard, notice also that the inference method to apply in the estimation of inequalities is not obvious. One could argue that the distance between observations and estimations should be linked to a measure of inequalities.

We would like to add that we see the empirical relevance of GAS model as an intriguing but encouraging outcome. It is both intriguing and encouraging for the same reason 
which is that, apparently, usual explanations of wage inequality are not required. Should GAS hypothesis be treated as an "as if" explanation? This is possible though individuals' efforts may reflect their investments in human capital, learning by doing, networking etc... 


\section{Concluding remarks}

Perhaps the main insight of this essay is that income inequality can result from the desire for (advantageous) inequality. This insight calls into question the precise meaning of studies showing that income inequalities "explain" life expectancy, mental health, toxicomania, obesity..16 It cannot be ruled out that all these social issues, including income inequalities, are driven by one common cause: the GAS attitude. We also showed that the GAS hypothesis has an empirical potential in replicating the observed wage dispersion.

In this conclusion we would like to note that our GAS model could be helpful in many other contexts where, whatever its form may be, a rat-race prevails, as, for instance, informative advertising ${ }^{17}$ with endogenously differentiated goods, or in the population density of cities where workers' on-the-job performance increases with their proximity to firms. Indeed, the proximity to a place of work can be regarded as a means of dealing with shocks, as with human capital in the Schultz/Nelson-Phelps view 18 Our analysis of GAS attitude raises different issues. As noted in the introduction, building a dynamic model with adaptative expectations would make the analysis more expressive. A limitation to our analysis is that incomes are assumed to be common knowledge. What is going on when incomes are private information? Why are tax returns public in some countries - like Norway where daily local newspapers report your neighbors' incomes - whereas they are secret in many others? And, still assuming imperfect information, what are the social consequences of the poor mimicking the rich, or hiding their poverty, and of the rich seeking to demonstrate to the poor that they are rich. Which goods are produced to help rich in their ostentation strategy? In France, Hermes offers a handbag which it advertises as reserved to women with the necessary age and elegance. Symmetrically, which goods do poor people use in their (paltry) attempt at cheating. Allowing for imperfect information would lead to build a two-stage game which would combine the social competition argument with the signalling argument 19 We leave these different questions for further investigation.

\footnotetext{
${ }^{16}$ See Wilkinson and Pickett (2010). Whatever their interpretation may be, the empirical results given by the authors are puzzling.

${ }^{17}$ Butters (1977) and Grossman and Shapiro (1984) are seminal papers.

${ }^{18}$ See Acemoglu and Autor (2012).

${ }^{19}$ See Danau and Gavrel (2015) for a first pass.
} 
We also assign to a companion paper the task of appraising the extent to which international disparities in income inequalities can be imputed to cultural specificities of GAS social rules.

\section{References}

Acemoglu, D., Autor, D., 2012. Lectures in Labor Economics. MIT press.

Arrow, K.J., 1975. Thornstein Veblen as an Economic Theorist. The American Economist 19(1), 5-9.

Burdett, K., Judd, K.L., 1983. Equilibrium Price Dispersion. Econometrica 51(4), 955-69.

Burdett, K., Mortensen, D.T., 1998. Wage Differentials, Employer Size, and Unemployment. International Economic Review 39(2), 257-73.

Butters, G.R., 1977. Equilibrium Distribution of Sales and Advertising Prices. Review of Economic Studies 44(3), 465-491.

Cahuc, P.,Postel-Vinay, F., 2005. Social Status and the Overworked Consumer. Annales d'Economie et de Statistique 78, 143-161.

Card, D., Mas, A., Moretti, Saez, E., 2012. Inequality at Work: The Effect of Peer Salaries on Job Satisfaction. American Economic Review 102(6), 2981-3003.

Clark, A.E., Oswald A.J., 1996. Satisfaction and Comparison Income. Journal of Public Economics 61(3), 359-381.

Danau, D., Gavrel, F., 2015. Keeping up with the Joneses as an Outcome of Going Ahead of the Smiths. One Simple Model for Two Behavioral Hypotheses. Mimeo, CREM, Normandy University, Caen and CNRS.

Duesenberry, J. S., 1949. Income, Saving and the Theory of Consumer Behaviour, Cambridge, Harvard University Press.

Easterlin, R., 1974. Does Economic Growth Improve the Human Lot? In Paul A. David et Melvin W. Reder, Nations and Households in Economic Growth: Essays in 
Honor of Moses Abramovitz, New York, Academic Press.

Friedman, D., Ostrov, D.N., 2008. Conspicuous Consumption Dynamics. Games and Economic Behavior 64(1), 121-145.

Gavrel, F., 2012. On the Inefficiency of Matching Models of Unemployment with Heterogeneous Workers and Jobs when Firms Rank their Applicants. European Economic Review 56(8), 1746-1758.

Gavrel, F., 2015. Participation, Recruitment Selection, and the Minimum Wage. Scandinavian Journal of Economics 117(4), 1281-1805.

Grossman, G.M., Shapiro, C., 1984. Informative Advertising with Differentiated Products. Review of Economic Studies 51(1), 63-81.

Hopkins, E., 2008. Inequality, Happiness and Relative Concerns: What Actually is their Relationship? Journal of Economic inequalities 6(4), 351-372.

Hopkins, E., Kornienko, T., 2004. Running to Keep in the Same Place: Consumer Choice as a Game of Status. American Economic Review 94(4), 1085-1107.

Ireland, N.J., 2001. Optimal Income Tax in the Presence of Status Effects. Journal of Public Economics 81, 193-212.

Kahneman, D., Krueger, A.B., Schkade, D., Stone A.A., 2006. Would You Be Happier If You Were Richer? A Focusing Illusion. Science 312(5782), 1908-1910. DOI: 10.1126/science.1129688

Leibenstein, H., 1976. Beyond Economic Man: A New Foundation for Microeconomics, Harvard University Press, Cambridge.

Mirrlees, J.A, 1971. An Exploration in the Theory of Optimum Income Taxation. Review of Economic Studies 38(2), 175-208.

Smith, A., 1776. An Inquiry into the Nature and Causes of the Wealth of Nations. London: Methuen and Co., Ltd., ed. Edwin Cannan, 1904. Fifth edition.

Ulph, D., 2014. Keeping up with the Joneses: Who Loses out? Economics Letters 
$125,400-403$.

Veblen, T., 1899. The Theory of the Leisure Class. An Economic Study of Institutions. Edition 1915 at Internet Archive.

Wilkinson R., Pickett, K., 2010. The Spirit Level. Why Equality is Better for Everyone. Penguin Books. U.K.

\section{Appendix}

\section{A. Equilibrium distribution $\Pi^{*}($.}

This appendix shows that $\Pi^{*}($.$) is continuous and that its support is connected and$ bounded above.

In a preliminary step, we show that $\Pi^{*}($.$) is continuous for k \geq 0$. Suppose it is not. This means that $\Pi^{*}\left(\right.$.) has at least one mass point $K_{i} \geq 0$ of probability $\phi\left(K_{i}\right)>0$. Suppose that a participant, $D$, who invests $K_{i}$ initially, deviates and decide on a higher investment $K_{i}+\varepsilon$. Since $\Pi\left(K_{i}^{+}\right)=\Pi\left(K_{i}\right)+\phi\left(K_{i}\right)$, we can deduce that, due to the continuity of $J($.$) and H($.$) , her utility will jump upwards, implying that K_{i}$ is not an optimum. This proves by contradiction that $\Pi^{*}($.$) is continuous.$

Next step shows that if the support of $\Pi^{*}($.$) , denoted by S^{*}$, contain two intervals $[A, B]$ and $[C, D]$ with $D>C>B>A \geq 0$, then it also contains the interval $[B, C]$. Suppose $S^{*}$ does not contain $[B, C]$. We then have $\Pi^{*}(k)<\Pi^{*}(B)$ for $A<k<B$, $\Pi^{*}(k)=\Pi^{*}(B)$ for $B \leq k \leq C$ and, $\Pi^{*}(k)>\Pi^{*}(C)=\Pi^{*}(B)$ for $C<k<D$. For all $k$ in the support, $U(k)=U^{*}$. This implies that $U(B)=U(C)=U^{*}$. As $\Pi^{*}(k)=\Pi^{*}(C)=\Pi^{*}(B)$ for all $k$ in $[B, C]$, we also have $J(B)=J(C)=J(k)$ in

the interval $[B, C]$. Since $H($.$) is strictly concave, U(k)=-k+J(B) H(k)$ is strictly concave. Consequently, $U(k)>U(B)=U^{*}$ for all $k$ in $] B, C$. This contradiction proves that the support of $\Pi^{*}($.$) is an interval [A, B]$ such that $0 \leq A<B \leq \infty$.

We can now show that the support of $\Pi^{*}($.$) is bounded above. Suppose it is not.$ Consequently, this support is an interval $[A, \infty$ [ with $A \geq 0$. For all $k>0, U(k) \leq$ $V(k)$. It results that $U(k)$ tends to $-\infty$ when $k$ tends to $+\infty$. As $U(0)>0$, this is a 
contradiction which sets that $S^{*}$ is bounded above.

In other words, $S^{*}$ is an interval $[A, B]$ with $0 \leq A<B<\infty$.

\section{B. Isomorphism between the distribution model $\Pi(k)$ and the ranking model $k(r)$}

If $\Pi($.$) is continuous and strictly increasing - like GAS equilibrium \Pi^{*}($.$) - the mapping$ between the ranking model, $k(r)$, and the distribution model, $\Pi(k)$, is a simple change in variables. This appendix shows how this mapping extends to piecewise continuous non-decreasing functions.

Namely, we show that any piecewise continuous c.d.f., $\Pi(k)$ translates into a piecewise continuous non-decreasing "ranking function" $k(r)$.

Let us first consider an interval $\left[k_{1}, k_{2}\right]$ on which $\Pi(k)$ is strictly increasing. On this interval $\Pi($.$) has a reciprocal k(r)=\Pi^{-1}(r)$. For all $r$ in $\left[r_{1}=\Pi\left(k_{1}\right), r_{2}=\Pi\left(k_{2}\right)\right]$, the share of individuals whose ranks are lower than $r$ is Prob[Rank $<r]=\operatorname{Prob}[$ Effort $\left.<\Pi^{-1}(r)\right]=\Pi\left(\Pi^{-1}(r)\right)=r$.

Let us now consider the case of a mass point, $K$, of $\Pi($.$) such that \Pi\left(K^{+}\right)=\Pi(K)+$ $\Phi(K)$, with $0<\Phi(K) \leq 1$. Remember that, according to our definition, $\Pi(K)=$ Prob[Effort $<K]$.

One can see that in this case, $k(r)$ is a constant equal to $K$ for all $r$ in the interval $\left[r_{3}=\Pi(K), r_{4}=\Pi(K)+\Phi(K)\right]$.

In this interval, the GAS multiplier, $J(\Pi(k(r)))$, is constant and equal to $J\left(r_{3}\right)$. This implies that

$$
\Sigma\left([k(.)]_{r_{3}}^{r_{4}}\right)=\int_{r_{3}}^{r_{4}}\left[-k(r)+J\left(r_{3}\right) H(k(r))\right] d r=\Phi(K)[-K+J(\Pi(K)) H(K)]
$$

Finally, we have to deal with holes in the support of $\Pi(k)$.

Suppose $\Pi($.$) is constant in an interval \left[k_{5}, k_{6}\right]$ while increasing in the left hand neighborhood of $k_{5}$ as well as in the right hand neighborhood of $k_{6}$. One can see that the 
rank associated with this interval is $R=\Pi\left(k_{5}\right)=\Pi\left(k_{6}\right)$. In addition, $k(R)=k_{5}$ and $k\left(R^{+}\right)=k_{6}$. In words, the ranking function $k($.$) is discontinuous at R$ and jumps upwards.

\section{Social optimum}

Due to the isomorphism between $\Pi($.$) and k($.$) , the social optimum can be defined as$ a ranking function $k^{S}(r)$ on $[0,1]$ which maximizes

$$
\Sigma(k(.))=\int_{0}^{1}[-k(r)+J(r) H(k(r))] d r
$$

It follows that, assuming that $-1+\mu H^{\prime}(0)>0$, as in Proposition $1, k^{S}(r)$ is determined by $-1+J(r)[d H(k(r)) / d k(r)]=0$ for all $r$ in $[0,1]$.

Since $H($.$) is strictly concave and J($.$) is strictly increasing, k^{S}(r)$ is strictly increasing. Consequently, the case in which the social optimum $k^{S}(r)$ would be constant on a subinterval of $[0,1]$ is clearly excluded. Indeed, since $J(r)$ is strictly increasing, the maximum of $\int_{r_{3}}^{r_{4}}[-k(r)+J(r) H(k(r))] d r$ with respect to $[k(r)]_{r_{3}}^{r_{4}}$ is higher than the maximum of $\left(r_{4}-r_{3}\right)\left[-K+J\left(r_{3}\right) H(K)\right]$ with respect to $K$. In words, assuming that $k(r)$ is constant on an interval $\left[r_{3}, r_{4}\right]$ with $\left(0 \leq r_{3}<r_{4} \leq 1\right)$ would generate a constraint on the planner's problem. The same holds for a discontinuity of $k(r)$. Since $k^{S}(r)$ is strictly increasing, the optimal distribution, $\Pi^{S}(k)$ is the reciprocal of $k^{S}(r)$. This implies that $\Pi^{S}(k)$ is strictly increasing in its (connected) support $\left[A^{S}=k^{S}(0), B^{S}=k^{S}(1)\right]$. Mass points and holes in the support of $\Pi^{S}(k)$ are not compatible with optimality. 